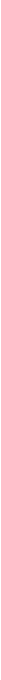

\title{
La máquina de hacer pastillas - The pill-making machine
}

Authors: Julieta Alcain, Nicolas Chiarante, Matías Zelaya

Submitted: 19. February 2022

Published:

Volume:

21. February 2022

Issue:

Affiliation:

Languages:

Keywords:

Categories:

DOI:

9

1

EGLC Journal, Buenos Aires, Argentina

Spanish, Castilian

Pharma, Supply, Patients, Market Price, Pills

News and Views, Medicine

10.17160/josha.9.1.804

\section{Abstract:}

Pharmaceutical companies do not set their prices based on production costs, but rather based on market price. In courtiers under development, this frequently limits the access of pharmaceutical products to people that need them but cannot afford it. Price mainly depends on the relationship between supply and demand, competition with other suppliers, and the level of 'freedom' with which pharmaceutical companies can operate. In the present article, Julieta Alcain, Nicolas Chiarante, and Matías Zelaya discuss in detail each of these variables, and how public production of medicines may secure their supply to every patient that may need them. Ilustrador: Marcos Montenegro Previous publication: 30/8/19 Link a la nota original: https://elgatoylacaja.com/la-maquina-de-hacer-pastillas/

\section{JOSHA Jouma a s semenes Humanities and Arts




\title{
La máquina de hacer pastillas - The pill-making machine
}

\author{
Julieta Alcain, Nicolas Chiarante, Matías Zelaya \\ llustrador: Marcos Montenegro \\ Fecha de publicación: 30/8/19 \\ Link a la nota original: https://elgatoylacaja.com/la-maquina-de-hacer-pastillas/
}

\begin{abstract}
:
Pharmaceutical companies do not set their prices based on production costs, but rather based on market price. In courtiers under development, this frequently limits the access of pharmaceutical products to people that need them but cannot afford it. Price mainly depends on the relationship between supply and demand, competition with other suppliers, and the level of 'freedom' with which pharmaceutical companies can operate. In the present article, Julieta Alcain, Nicolas Chiarante, and Matías Zelaya discuss in detail each of these variables, and how public production of medicines may secure their supply to every patient that may need them.
\end{abstract}


Aaah, las drogas. Qué sería de la humanidad sin ellas. Y no hablamos de los cartoncitos de colores o los brownies mágicos que se consumen con fines 'recreativos' por sus efectos psicoactivos, sino de las que consumimos para sobrevivir a enfermedades e infecciones: analgésicos, antibióticos, antihipertensivos, hipoglucemiantes, y un largo etcétera. La cantidad de medicamentos de los que dependemos es enorme, y la cantidad de plata (pública y privada) invertida en la compra de esos medicamentos es monstruosa. ¿Realmente es necesario semejante gasto? ¿Podemos ahorrar en medicamentos?

Quizá la primera respuesta que se nos ocurra sea por supuesto que no, con la salud no se juega. Ahorrar implicaría comprar menos o más barato, pero los medicamentos son lo que en economía se llama 'bienes de demanda inelástica', es decir que, sin importar cuánto cueste y cuánta plata tengamos, si el médico lo prescribe intentaremos comprarlo. Además, sincerándonos, si en la farmacia nos ofrecen dos medicamentos con los mismos componentes, pero uno cuesta la mitad de plata que el otro, ¿no es probable que miremos con desconfianza el remedio barato? Tendemos a pensar que podemos recortar gastos comprando una segunda, tercera o cuarta marca de galletitas o pantalones, pero ahorrar unos pesos en el antibiótico es más complejo, no se nos ocurre arriesgarnos a terminar con algo peor o, por lo menos, a no curarnos nunca de la infección.

Las empresas productoras de fármacos lo saben. Por eso, si bien contemplan una inversión en investigación y desarrollo, en nuestro país la cantidad de dinero invertido en I+D es muy poca en comparación con lo que invierten en marketing. No es casual: el desarrollo de nuevos fármacos requiere una inyección considerable de dinero y no promete resultados asegurados. En su lugar, la inversión fuerte de esta industria está dirigida a presentarse como la empresa líder, la que da confianza. Y a contratar legiones de visitadores médicos. Porque por más que en 2002 se sancionó la Ley de Genéricos (que obliga a los médicos a prescribir los medicamentos por droga y no por marca o nombre comercial, y a los farmacéuticos a ofrecer alternativas de diferentes precios), un vacío legal en la reglamentación de dicha ley permite que se 'sugiera' una marca determinada en la receta y que en la farmacia te den lo que la receta indica. $Y$ el paciente, desinformado o mal informado, suele llevarse la marca recetada por el médico. Mientras tanto, los laboratorios trabajan para inclinar en su favor las preferencias de los médicos, ofreciendo premios y regalos a profesionales de la salud en base a la cantidad de medicamentos prescriptos. 


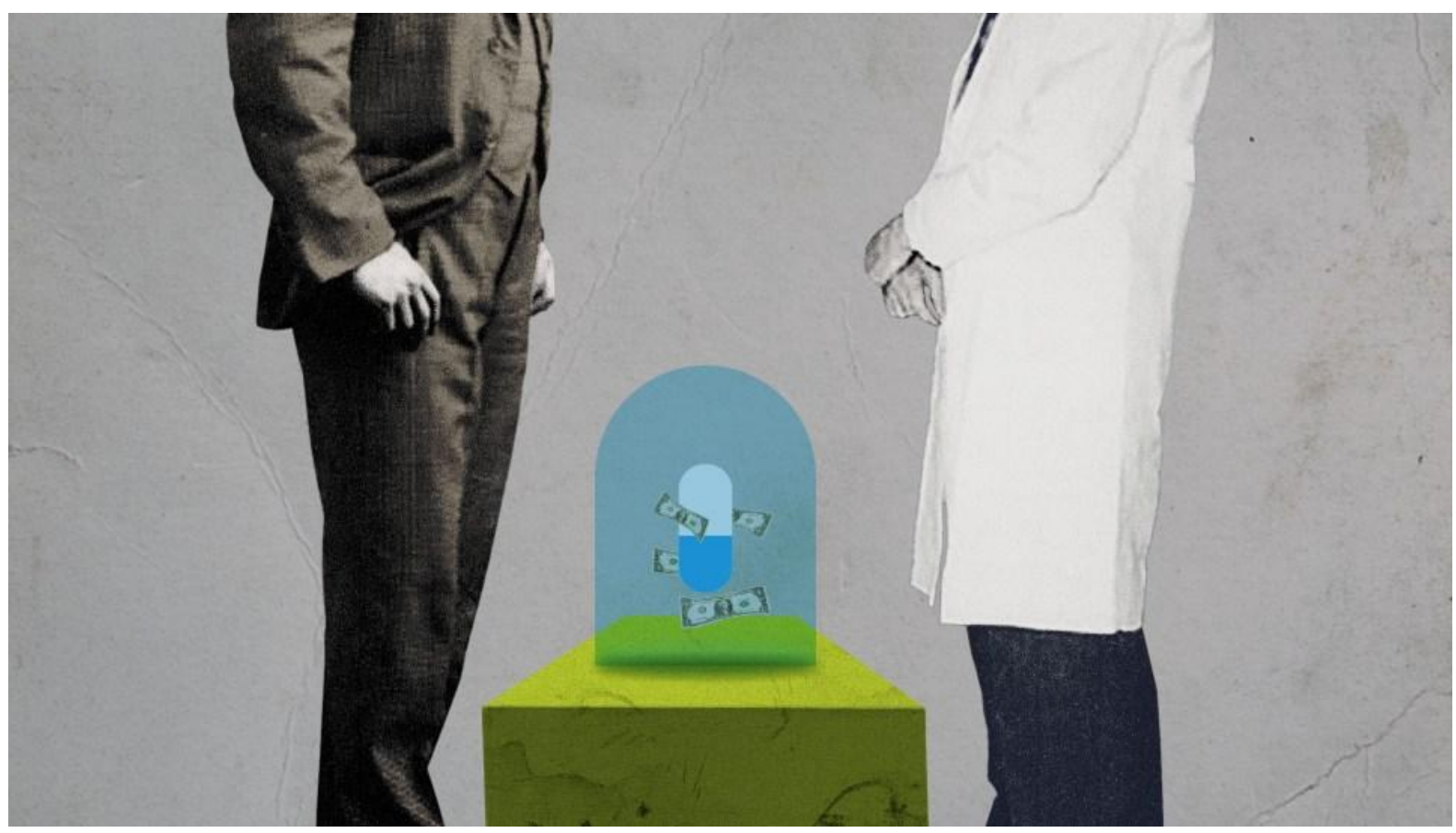

\section{Comprimido de historia}

La ley de genéricos se sancionó en el marco de una fuerte crisis sanitaria directamente relacionada con la crisis económica y política que sufrió Argentina a comienzos de este milenio. La altísima desocupación generó un aumento de la población sin cobertura de salud, demanda que fue absorbida por los hospitales públicos. En ese momento, el consumo de medicamentos se comportaba de manera muy similar al de cualquier otro bien costoso: los consumía quien los podía pagar. La situación era alarmante. Casi la mitad de las personas más pobres del país no había accedido a medicamentos recetados durante el 2001 (de hecho, en 10 años había disminuido a la mitad el consumo de medicamentos, y no precisamente porque estuviéramos menos enfermos).

En el medio de la declarada emergencia sanitaria nacional, el Gobierno Nacional, a través de su ministro de salud, Ginés González García, formuló en el año 2002 lo que se llamó 'Política nacional de medicamentos'. Una parte de las estrategias, como el caso de la ley de genéricos, se dirigían a mejorar las condiciones de acceso de la población que sí tenía capacidad de compra o que, por lo menos, contaba con un sistema de cofinanciamiento de los medicamentos. Por otra parte, para el aluvión de personas sin dinero para comprarlos, se implementó el Programa Remediar de provisión pública de medicamentos: el Ministerio de Salud compraba mediante licitación los remedios prioritarios y los repartía en el primer nivel 
de atención (salitas, dispensarios o centros de salud barriales), donde las personas podían retirarlos sin cargo.

El Plan Remediar y la ley de genéricos tuvieron un efecto colateral positivo para la industria nacional: al comprar los medicamentos de las industrias farmacéuticas locales, las levantó de la crisis económica en la que se habían sumido durante los años '90. Pero junto con la recuperación de la capacidad productiva de estas empresas, se generó un fuerte aumento en los precios de los medicamentos. En parte esto se debió al 'efecto murciélago': las marcas que, en un principio, eran más baratas, rápidamente se 'colgaron' del precio de las marcas líder, e incluso, en algunos casos, acabaron siendo más caros los medicamentos locales que sus versiones originales, provenientes de algún laboratorio del exterior. Esto también tuvo que ver con lo que no se consiguió desde el Estado: negociar mejores precios para los medicamentos nacionales aprovechando su rol como cliente con un inmenso poder de compra. Era (y sigue siendo) tal el volumen de compra del Estado que generó una importante presión presupuestaria: se estaba gastando mucha plata en remedios.

\section{Un monstruo grande y pisa fuerte}

Mucha de la plata que se gasta en medicamentos es, en realidad, resultado de sobreprecios: las farmacéuticas, aunque no son las únicas, no establecen los precios en base a cuánto les cuesta producir, sino en base al precio que pueden conseguir en el mercado. La formación de precios depende principalmente de tres cuestiones: la relación entre oferta y demanda, la competencia con otros proveedores y los niveles de 'libertad' con los que pueden comercializar. Veamos entonces cómo afectan estos tres factores al precio de los medicamentos.

En primer lugar, la relación entre oferta y demanda es bastante despareja cuando del lado de la demanda hay poca flexibilidad. Y eso es precisamente lo que pasa cuando hablamos, como en este caso, de un consumo asociado a una necesidad básica, que no se puede simplemente reemplazar por otro producto y, sobre todo, cuando hay un Estado que debe garantizar el acceso. Y decimos 'debe' en un sentido más legal que moral: el acceso a la salud es un derecho consagrado en la Constitución Nacional Argentina y en tratados internacionales, por lo que se trata de una obligación. Ir a ver al médico y llevarse una receta pero no poder comprar los medicamentos prescriptos, ¿cuenta como acceso a la salud?

Por otro lado, la competencia tampoco regula demasiado los precios cuando los compradores no saben que hay más de un proveedor. Recordemos que fue necesaria una ley para que se garantice esa información a quienes compran medicamentos. Pero esa ley no siempre es respetada (de hecho, aunque desde hace tiempo no hay datos oficiales, las 
más recientes consultas y relevamientos no oficiales indican que más de la mitad de las recetas son ilegales, dada la falta de nombre genérico de la droga). Además, muchos compuestos que fueron desarrollados por la industria privada están protegidos por patentes que, como compensación por el gasto de desarrollo del producto, le otorgan a la empresa que posee esa patente el monopolio de su comercialización por veinte años, impidiendo la competencia con productos de otras empresas a precios más bajos.

La tercera cuestión es que la libertad de mercado puede ser, al menos parcialmente, limitada por regulaciones públicas. Y ahí entra en juego la capacidad de presión de un sector que ha hecho muestra de su poder en diferentes episodios de la historia argentina.

En los años '60, Arturo Illia (ex presidente argentino y en su momento médico de formación) advertía que los medicamentos estaban muy sobrevaluados. Siendo presidente, impulsó en 1964 lo que se conoce como Ley Oñativia (en honor a su ministro de Salud Pública): dos leyes mediante las cuales se obligaba a los laboratorios a presentar declaraciones juradas indicando los costos de producción y justificando los precios. Además, se establecía un límite a la publicidad y a los pagos al exterior en concepto de compra de insumos y se depuraron los registros farmacéuticos, eliminando un $45 \%$ de formulaciones registradas por considerar que no cumplían con los estándares requeridos. Las medidas del gobierno de Illia contra las empresas multinacionales le costaron bastante caro. El Club de París hizo llegar al ministro de economía y al mismo presidente el mensaje de que las leyes que regulaban el mercado farmacéutico harían peligrar la renegociación de la deuda externa argentina, así como futuros financiamientos. En 1966 un golpe militar derrocó al gobierno de Arturo Illia, y al año siguiente las leyes de Oñativia fueron derogadas por el presidente de facto Juan Carlos Onganía. Las políticas de liberalización y desregulación tuvieron su momento de oro en los '90, cuando el decreto 2.284 del año 1991, entre otras medidas, autorizó la venta de medicamentos sin receta fuera de farmacias, y mayores facilidades para la importación de medicamentos elaborados (esto fue revertido en el 2009 al sancionarse la Ley 26.567, que dispone que "la preparación de recetas, la dispensa de drogas, medicamentos, incluidos los denominados de venta libre y de especialidades farmacéuticas, cualquiera sea su condición de expendio, sólo podrán ser efectuadas en todo el territorio de la Nación, en farmacias habilitadas").

\section{Hecho en casa}

Aunque el panorama parezca bastante negro, y se pueda pensar que no hay manera de ganarle a la industria farmacéutica (una de las que más dinero mueven a nivel mundial), hay alternativas: existen sociedades del Estado (LIF, Laboratorio Industrial Farmacéutico S.E., LEM, Laboratorio de Especialidades Medicinales S.E., Laboratorios Puntanos S.E., entre otros) cuyo objetivo es producir y abastecer a los hospitales y salitas de estos remedios. 
Reciben el nombre de laboratorios de Producción Pública de Medicamentos (PPM). Como el cliente principal son los Ministerios/Secretarías de Salud, que también es el Estado, la venta de los medicamentos se hace a precio de costo (con un margen de ganancia justo que permita mantener la producción en las plantas elaboradoras). Además, es una excelente herramienta de control de precios, porque fija lo que se llaman 'precios testigo' que sirven como presión para bajar los precios de las otras marcas e indican cuánto cuesta realmente producir un medicamento. Valga el siguiente ejemplo.

En el año 2009 el Ministerio de Salud argentino lanzó una campaña nacional contra la hidatidosis, una de las llamadas 'enfermedades desatendidas en poblaciones postergadas' según la Organización Panamericana de la Salud. Dicho de otro modo, uno de esos bichos que se la agarran especialmente con poblaciones de bajos recursos (como el Chagas y otras parasitosis). Para tratar esta enfermedad, se requiere un medicamento llamado albendazol, que el Ministerio solicitó a la empresa Novartis, y fue presupuestado en \$22 por comprimido. No satisfecho con el precio ofrecido por la multinacional, el Ministerio solicitó entonces un presupuesto al Prozome (actualmente llamado Profarse, laboratorio estatal localizado en la provincia de Río Negro), que lo ofreció a la irrisoria suma de \$0,50 por comprimido. ¿Qué eran los otros $\$ 21,50$ extra que cobraba Novartis? Si bien es lógico que entre los costos de producción de los medicamentos se compute la inversión en desarrollo, y que parte de la ganancia se puede y debería reinvertir en más investigación, es cuanto menos inquietante que el $98 \%$ del precio del fármaco no esté relacionado con el costo de producción. Novartis pretendía ganar $\mathbf{4 3}$ veces más de lo que en verdad costaba producirlo y comercializarlo. Prozome ganó la licitación y entregó 100.000 comprimidos, permitiéndole al Estado gastar considerablemente menos.

Los laboratorios de Producción Pública de Medicamentos (PPM) funcionan hace muchos años en Argentina. Al menos desde la Primera Guerra Mundial, cuando, en parte por la escasez de insumos importados desde Europa, se tomó la decisión de producir algunos de ellos en el país para satisfacer la demanda pública. Así nació el Instituto Bacteriológico (hoy denominado ANLIS-Malbrán) a principios del siglo XX, que utilizaba como materia prima las glándulas provenientes de los mataderos municipales (cedidas de forma gratuita) para la fabricación de sueros, vacunas y otros productos médicos que distribuía a los hospitales públicos. Le siguieron otros, que fueron surgiendo en diferentes zonas del país, bajo control de diferentes esferas del Estado, para atender la demanda local a precios razonables.

La provincia de Santa Fe es un caso paradigmático. Abastece el $94 \%$ de los medicamentos para atención primaria de la salud y el $66 \%$ de los medicamentos del sistema público provincial con la producción de sus tres laboratorios de PPM: el LIF (Laboratorio Industrial Farmacéutico), el LEM (Laboratorio de Especialidades Medicinales) y la planta piloto que funciona en la Facultad de Ciencias Bioquímicas y Farmacéuticas de la Universidad Nacional 
de Rosario. Y la diferencia es tanta que la provincia de Santa Fe paga los medicamentos un 32\% menos que la Nación. Uno podría suponer que es al revés: que el Estado nacional, que compra 40 veces más medicamentos que esta provincia, tiene más poder como cliente para negociar y conseguir bajos precios. Pero más allá del volumen de compra, que evidentemente debería ser un factor clave en la negociación, esta diferencia de precios parece deberse en gran parte a la implicancia que tienen los laboratorios públicos en Santa Fe.

En total hay 34 unidades de PPM en el país, distribuidas en varias provincias y con diferentes dependencias: provincial, municipal e incluso universitaria. Sólo 12 de ellas tienen habilitación de la Administración Nacional de Medicamentos, Alimentos y Tecnología Médica (ANMAT), y este dato no es menor, porque es esta habilitación la que permite que los productos salgan de las provincias a las rutas nacionales para distribuirse por el país. Los laboratorios que no la poseen pueden producir fármacos y especialidades médicas únicamente para su distribución provincial. Si se pudiera avanzar en realizar las reformas edilicias necesarias para que los laboratorios puedan ser habilitados por ANMAT, y con una coordinación nacional eficiente, se podría organizar la producción de manera estratégica para poder pasar a escalas mayores a las provinciales. Por poner sólo un ejemplo, los laboratorios podrían especializarse en la fabricación de un tipo de medicamentos en particular, disminuyendo los tiempos de limpieza de los equipos y el gasto de dinero en insumos, originando un aumento en la capacidad de producción y optimizando el uso de los recursos disponibles.

Aunque la producción pública de medicamentos fue declarada de interés nacional en 2011 -y por ley se creó en 2014 la Agencia Nacional de Laboratorios Públicos (ANLaP), que es el organismo que nuclea y coordina a los laboratorios públicos- en la actualidad esta es una política que no ocupa un lugar prioritario ni estratégico. La financiación de los laboratorios públicos es intermitente y escasa, lo que genera que las leyes que estimulan la expansión del sector no se cumplan del todo. Así, se impide que los laboratorios puedan ampliar su producción y afrontar con soltura gastos ordinarios como mantenimiento edilicio e instalaciones, validación anual externa de equipamiento y adquisición de materias primas, reactivos e insumos (todo esto a precio dólar); así como también gastos extraordinarios como reparación de equipamiento. En los últimos años, el presupuesto destinado a la ANLaP no sólo no fue ajustado respecto a la inflación, sino que incluso disminuyó nominalmente desde su creación en el año 2015. Pero los laboratorios estatales no necesitan únicamente más plata, sino también que se les garantice la demanda de su producción. $Y$ el Estado continúa adquiriendo mayoritariamente en el mercado privado medicamentos que podría comprar, mucho más baratos (como ya vimos), a laboratorios estatales. Por ejemplo, el Instituto Biológico de La Plata "Dr. Tomás Perón", habilitado por ANMAT, tiene la infraestructura y el conocimiento para producir la vacuna BCG contra la 
tuberculosis (la cual es de referencia para América Latina según la OMS). Sin embargo, el Estado sigue importándola. Esta última es una manera indirecta de paralizar la producción pública de medicamentos, porque aunque los laboratorios pudiesen producirlos a gran escala, si nadie los demanda, esa producción lógicamente comienza a disminuir hasta desaparecer.

La producción pública de medicamentos no es sólo una herramienta eficaz para el control de los precios: también es un pilar fundamental de nuestra soberanía sanitaria. La industria farmacéutica es conocida por sus prácticas monopólicas y oligopólicas. Y por no estar enfocada necesariamente al cuidado de la salud, sino más bien de su ganancia. Es cierto que la industria farmacéutica privada cumple un rol importante en la innovación, y es cierto que para seguir cumpliendo ese rol debe ser rentable, pero depender exclusivamente de ella no parece la mejor opción. Para las farmacéuticas privadas el medicamento es una mercancía, un producto con el que se busca únicamente generar ganancias. Esta concepción del producto farmacéutico explica (además de su altísimo precio en algunos casos) por qué la industria privada no suele producir medicamentos que no generan ganancias (denominados 'huérfanos'), como los tuberculostáticos. Al ser medicamentos antiguos no tienen patente, lo que hace que sean muy baratos y (al no ser tampoco de consumo masivo) no produzcan grandes ganancias, y por esto no hay producción privada de estos antibióticos. Si no existiera su producción pública en laboratorios como el de Talleres Protegidos de la Capital Federal, o del PROFARSE en Río Negro, el $100 \%$ de los tuberculostáticos deberían ser importados al doble del costo de producción local.

Otro ejemplo para nada menor es el del misoprostol: en algunos laboratorios públicos, como en el LIF de Santa Fe, se está desarrollando y trabajando para escalar la producción pública de esta droga, cuyo precio en 2019 ronda los $\$ 5800$ por 16 comprimidos de la marca privada (con un $75 \%$ de aumento respecto de 2018). El laboratorio público consiguió producir misoprostol a menos del $10 \%$ de su valor comercial. Es importante aclarar que, incluso dejando de lado el debate por la legalización de la interrupción voluntaria del embarazo, el misoprostol es una droga clave para completar abortos espontáneos que acarrean riesgos de infecciones, con lo cual su elevado precio implica un impedimento para el acceso a la salud de las mujeres, incluso en casos que se encuentran dentro de los márgenes de la ley actual. De hecho, una caja de Oxaprost cuesta, a mayo de 2019, el equivalente al $47 \%$ del salario mínimo vital y móvil.

En Argentina, la Constitución Nacional (desde la reforma de 1994), establece en el artículo 42 que los ciudadanos tienen derecho a la protección de su salud y seguridad. Además, al otorgar jerarquía constitucional a once pactos y tratados internacionales de derechos humanos, se establece que la salud es un derecho. El artículo 12 del Pacto Internacional de Derechos Económicos, Sociales y Culturales (PIDESC), explicita que "los Estados Partes en 
el presente Pacto reconocen el derecho de toda persona al disfrute del más alto nivel posible de salud física y mental".

Por lo tanto, si la salud es un derecho, los medicamentos no deben ser una mercancía sino un bien social. Esto significa que el acceso a los medicamentos debe estar garantizado (por supuesto, junto con la atención médica y una alimentación saludable). Y una forma de garantizar este derecho, tanto en épocas de brócolis gordos como en épocas de vacas flacas, es asegurándonos de que su provisión no dependa de la lógica de un grupo de empresas cuya prioridad es ganar lo más que puedan. Por eso (y hasta tanto no se nos ocurra una idea mejor), para encontrar una alternativa a los tremendos sobreprecios que imponen las farmacéuticas privadas, la producción pública de medicamentos como política de Estado podría ser la apuesta necesaria para garantizar drogas para todos y alcanzar una verdadera soberanía a nivel sanitario. 


\section{Referencias}

Mariana Mazzucato. The entrepreneurial state. Revista digital "Renewal”, Vol 19, num 3/4 2011.

Hernán Marmurek, Atrapados por el marketing. Revista Soberanía Sanitaria.

Guillermo Martín Santos. Tecnologías para la solución de problemas sociales. Alcances y restricciones de la producción pública de medicamentos en Argentina. Aprendizajes, dinámicas problema-solución y alianzas socio-técnicas. Universidad Nacional de Luján. 2017.

Martín Isturiz, "Producción estatal de medicamentos". Revista Industrializar Argentina, Año 12, Num 23. 2014.

Antonio Ugalde, Núria Homedes. "Medicamentos para lucrar. La transformación de la industria farmacéutica”. Salud Colectiva, vol. 5, núm. 3, septiembre-diciembre, 2009, pp. 305-322. Universidad Nacional de Lanús, Buenos Aires, Argentina

Ministerio de Salud, Presidencia de la Nación. “El programa Remediar. Gestión y resultados de un modelo innovador en APS". Marzo 2006. Buenos Aires, Argentina.

Karina Inés Ramacciotti y Lucía Romero. La regulación de medicamentos en la Argentina (1946-2014). Revista CTS, nº 35, vol. 12, Junio de 2017 (pág. 153-174) 